\title{
Effects of Androgen Deprivation Therapy on Blood Lipids and Fasting Blood Glucose in Patients with Prostate Cancer
}

\author{
(D) Mehmet Yarış \\ Genesis Private Hospital, Clinic of Urology, Diyarbakır, Turkey
}

\begin{abstract}
Objective: This study aimed to investigate the changes in the lipid profiles and fasting blood glucose (FBG) values of patients with prostate cancer, who underwent bilateral orchiectomy or started to use luteinizing hormone-releasing hormone (LHRH) agonists and additionally received anti-cyproterone acetate or bicalutamide, and compare these changes between alternative treatments.

Materials and Methods: This retrospective study included 66 patients with complete data. Patients' age, prostate-specific antigen values, prostate volumes, clinical stages, pathology results, type of androgen deprivation therapy, and total cholesterol (TC), low-density cholesterol, high-density cholesterol, triglyceride, and FBG values before and at 6 months after treatment were recorded. Metastatic was determined in 37 patients, whereas 26 had locally advanced and 3 had localized prostate cancer.

Results: The evaluation of all patients revealed an increased FBG $(p=0.010)$ and low-density cholesterol $(p=0.012)$ values was significant. The comparison of patients who underwent orchiectomy and those taking LHRH agonists revealed no difference between the two groups. The difference in the TC values was statistically significant between the cyproterone acetate and bicalutamide groups $(p<0.001)$.
\end{abstract}

Conclusion: Bicalutamide significantly increases TC compared to cyproterone.

Keywords: Antiandrogens, hormonotherapy, lipid profiles, prostate cancer

\section{Introduction}

Prostate cancer is one of the most common cancers among males (1). Androgen deprivation therapy (ADT) has been frequently used to prolong survival and relieve symptoms in patients with prostate cancer. ADT forms the basis of treatment, especially in relapsed and metastatic prostate cancer (2), as well as adjuvant therapy in high-risk localized and locally advanced diseases $(3,4)$. ADT can be surgically (bilateral orchiectomy) and medically [luteinizing hormone-releasing hormone (LHRH) agonist-antagonist and antiandrogens] applied (2). ADT provides significant clinical benefits; however, it is associated with an increased risk of diabetes and cardiovascular diseases and a wide variety of complications, such as hot flashes, anemia, sexual dysfunction, body composition changes, osteoporosis, and fractures $(5,6,7)$. Some complications negatively affect the quality of life, whereas others may induce serious problems, including sudden cardiac death and myocardial infarction (8). Prostate cancer has a favorable prognosis, thus care should be taken when planning ADT due to its complications and patients should be followed up more closely. This study aimed to investigate the changes in the lipid profiles and fasting blood glucose (FBG) values of patients with prostate cancer, who underwent bilateral orchiectomy or started to use LHRH agonists and additionally received anti-cyproterone acetate or bicalutamide and compared these changes between alternative treatments.

\section{Materials and Methods}

This retrospective study reviewed the files of 98 patients who were diagnosed with prostate cancer in our clinic and started LHRH agonists or underwent ADT after bilateral orchiectomy in 2014. The hospital patient information system and e-nabız (Personal Health System of Turkey) were screened to record the patients' age, prostate-specific antigen (PSA) values, prostate volumes, clinical stages, pathology results, type of ADT applied, and total cholesterol (TC), low-density cholesterol (LDL), highdensity cholesterol (HDL), triglyceride (TG), FBG values before and at 6 months after treatment. This study included 66 patients with complete data, wherein 37 had metastatic, 26 had locally advanced, and 3 had localized prostate cancer. Patients with localized diseases constituted the high-risk group that did not

Cite this article as: Yarış M. Effects of Androgen Deprivation Therapy on Blood Lipids and Fasting Blood Glucose in Patients with Prostate Cancer. Bull Urooncol 2022;21(1):5-9 
accept surgery or radiotherapy. Maximal androgen blockade (MAB) was applied to 61 patients, whereas only LHRH analogs were initiated in 5 patients. Bilateral orchiectomy was performed in 25 patients and LHRH analogs were started in 41 patients. Among the patients receiving $M A B$, cyproterone acetate was started in 21 and bicalutamide in 40 . The general characteristics of patients are presented in Table 1.

\section{Statistical Analysis}

Statistical analyses were performed using the Statistical Package for the Social Sciences version 17.0 software package. The normal distribution of variables was examined using histogram graphics and the Kolmogorov-Smirnov test. Mean, standard deviation, and median values were used to present descriptive statistics. Changes in measured values were compared between the groups with the repeated-measure analysis and within each group using the Wilcoxon test. Results with a p-value of $<0.05$ were considered statistically significant.

Approval was obtained from the Ethics Committee of Diyarbakır Gazi Yaşargil Training and Research Hospital with the date and number 772/29.05.2021.

\begin{tabular}{|c|c|c|c|}
\hline & & $\mathbf{n}$ & $\%$ \\
\hline \multirow{3}{*}{ Clinical stage } & Localized & 3 & 4.55 \\
\hline & $\begin{array}{l}\text { Localized } \\
\text { advanced }\end{array}$ & 26 & 39.39 \\
\hline & Metastatic & 37 & 56.06 \\
\hline \multirow{2}{*}{ ADT } & ox & 25 & 37.88 \\
\hline & LHRH & 41 & 62.12 \\
\hline \multirow{5}{*}{ Treatment subgroup } & OX + cyproterone & 12 & 18.18 \\
\hline & $\begin{array}{l}\mathrm{OX}+ \\
\text { bicalutamide }\end{array}$ & 14 & 21.21 \\
\hline & $\begin{array}{l}\text { LHRH + } \\
\text { cyproterone }\end{array}$ & 9 & 13.64 \\
\hline & $\begin{array}{l}\text { LHRH + } \\
\text { bicalutamide }\end{array}$ & 26 & 39.39 \\
\hline & LHRH & 5 & 7.58 \\
\hline \multirow{3}{*}{ Antiandrogen } & Cyproterone & 21 & 31.82 \\
\hline & Bicalutamide & 40 & 60.61 \\
\hline & None & 5 & 7.58 \\
\hline
\end{tabular}

Table 2. Comparison of the pre-treatment and sixth-month values of all patients

\begin{tabular}{|l|l|l|l|}
\hline & Before treatment & Sixth month & p-value \\
\hline LDL & $123.59 \pm 33.89$ & $134.53 \pm 50.88$ & $\mathbf{0 . 0 1 2}$ \\
\hline HDL & $46.82 \pm 5.33$ & $52.07 \pm 47.45$ & 0.064 \\
\hline TC & $190.80 \pm 2.37$ & $199.09 \pm 47.53$ & 0.301 \\
\hline TC & $133.67 \pm 57.79$ & $133.85 \pm 63.53$ & 0.971 \\
\hline FBG & $105.22 \pm 37.29$ & $112.25 \pm 48.99$ & $\mathbf{0 . 0 1 0}$ \\
\hline
\end{tabular}

Wilcoxon test, LDL: Low-density cholesterol, HDL: High-density cholesterol, TC: Total cholesterol, TG: Triglyceride, FBG: Fasting blood glucose

\section{Results}

The mean and standard deviation values of age, PSA, and prostate volume of patients were $72.05 \pm 9.91$ years, $96.47 \pm 133.44$, and $55.55 \pm 28.03$, respectively. The changes in the $L D L, H D L, T C$, TG, and FBG values were analyzed before and at the sixth month of treatment. Accordingly, the evaluation of all patients revealed a significant increase in the LDL and FBG values. However, no significant changes were observed in the $\mathrm{HDL}, \mathrm{TC}$, and TG values (Table 2).

The comparison of patients who underwent orchiectomy $(n=25)$ and those who received LHRH $(n=41)$ for prostate cancer treatment in two separate groups revealed no significant difference. Additionally, changes in the measured values were compared within the orchiectomy and LHRH groups. No significant changes were observed in the LHRH group, whereas the LDL and FBG values significantly increased in the orchiectomy group (Table 3).

Changes in the measured values of patients were also compared between the cyproterone acetate (Androcur $($ )), bicalutamide (Casodex®), and non-antiandrogen (non-ADT) groups, which revealed no significant differences. The examination of changes within the groups revealed increased $L D L, H D L$, and $T C$ in the bicalutamide group, a decreased TC value, and an increased FBG value in the non-ADT group (Table 4).

Five different subgroups of treatment were applied to the patients, of which statistical comparisons were made; however, their results were excluded in this manuscript due to the small number of patients in each subgroup.

\section{Discussion}

ADT benefits have been well accepted for selected patients. Over the last few decades, ADT has substantially increased; however, relatively little attention has been paid to its side effects (9). Males with prostate cancer have higher non-cancer mortality rates than those in the general cancer population, and some of the non-cancer deaths may be associated with treatment (10). Metabolic changes are frequently observed in males undergoing ADT, which may lead to an increased risk of type 2 diabetes, cardiovascular diseases, and metabolic syndrome, and may contribute to increased non-cancer mortality (11). Many studies in the literature discussed ADT without details concerning the drug or treatment methods. Thus, this study investigated the difference between different treatment alternatives in terms of lipid profile or FBG. Differences were determined between the antiandrogen groups (Table 4). Considering the possibility of serious side effects of such drugs, care should be taken in drug treatment selection and our related findings were discussed in detail.

Many publications investigated the effect of ADT on the lipid profile of patients and reported different results. However, the majority of the work suggests that ADT increases TC $(12,13,14,15,16,17,18,19)$. The prospective study of Smith et al. (20) revealed no significant changes in the TC value after 6 months of ADT. Contrarily, a review of 15 studies published by Wolny-Rokicka et al. (21) in 2019 reported that ADT significantly increased TC. The current study evaluated all patients and 
revealed no differences in TC changes. Additionally, no significant difference was found between the orchiectomy and LHRH groups in the changes in TC. No increase was observed in TC in the cyproterone group, whereas a significant increase was found in the bicalutamide group, with a statistically significant level. To the best of our knowledge, no literature study had compared the changes in the lipid profile of antiandrogens. Therefore, our findings were compared with the results obtained for bicalutamide and cyproterone in previous studies. Studies that used cyproterone revealed no significant changes $(22,23)$ or a decrease in TC $(24)$. Studies that used bicalutamide have revealed an increased TC $(15,25)$. Our results seem to be consistent with the literature. Studies that examine the effect of ADT on the HDL level offer contradictory results. Studies reported that ADT increases HDL $(12,13,14,15,17,19)$, whereas others indicated no changes in HDL with ADT application $(16,18,20)$. Some studies revealed that ADT decreases HDL (26). A review by Wolny-Rokicka et al. (21) reported no significant changes in HDL. Our study evaluated all patients and revealed no significant changes in HDL. No significant difference was determined between the orchiectomy and LHRH groups in $\mathrm{HDL}$ changes. A significant increase was determined in $\mathrm{HDL}$ in the bicalutamide group, whereas no increase in the cyproterone group, without statistically significant differences between these two groups. Studies in the literature revealed that cyproterone reduces HDL $(22,23,24)$. However, no significant changes were observed in $\mathrm{HDL}$ in studies that use bicalutamide $(15,25)$. Therefore, our findings are not consistent with the literature.

Data obtained from research that examined the effects of ADT on LDL level are also conflicting. Some researchers report that ADT increases $\operatorname{LDL}(12,13,14,16,19,26)$, whereas others suggest no changes in $\operatorname{LDL}(15,20,22)$. Additionally, other studies revealed that $\operatorname{LDL}$ decreases with $\operatorname{ADT}(22,23,24,25,26)$. WolnyRokicka et al. (21) reported no significant change in LDL in their meta-analysis. The current study evaluated the whole sample and revealed a significantly increased LDL. The treatment group evaluation revealed a significantly increased LDL in the orchiectomy group but revealed no increase in the LHRH group, and the difference between the two groups was not statistically significant. Additionally, LDL significantly increased in the bicalutamide group but did not significantly change in the cyproterone group, without a significant difference between these two groups. Studies in the literature revealed that cyproterone reduces LDL (24) or without significant changes $(22,23)$, whereas an increase in bicalutamide $(15,25)$.

Studies that examine the effect of ADT on the TG level report either an increase $(13,15,19,22,26)$ or no changes $(14,16,17,18,20)$. Wolny-Rokicka et al. (21) reported that the TG value increased with ADT application. Our study revealed no significant changes in TG as evaluated in all patients together or different treatment groups were examined.

The literature review revealed that male hypogonadism is accompanied by an increased risk of insulin resistance and diabetes mellitus $(27,28)$. A meta-analysis of 43 studies involved 6,427 males and revealed significantly lower total testosterone levels in males with type 2 diabetes than those without diabetes (29). Males with prostate cancer who take ADT are excellent examples of hypogonadism; therefore, they may also have insulin resistance and hyperglycemia. Conflicting results were reported in the literature concerning the effect of ADT on FBG. A prospective study of 133 patients by Bo et al. (12) compared patients who received ADT with those who underwent radical prostatectomy and did not receive any other treatment, as well as with a completely healthy group. The group that received ADT revealed no changes in FBG at the third month compared to the other two groups, whereas a significant difference was found at 12 months. A multicenter, prospective study conducted by Morote et al. (13) with 310 patients revealed a significantly increased FBG level at 6 and 12 months. A prospective study by Gagliano-Jucá et al. (14) included 73 patients and compared patients with prostate cancer using ADT to those with localized prostate cancer who had not received hormonal therapy. The authors examined the values of patients at the baseline, 6 , 12 , and 24 weeks and reported that while insulin resistance significantly increased in the ADT group, FBG did not significantly change. A study by Yannucci et al. (15) reviewed the data of 1,102 patients (obtained from the patient pool of a prospective study), measured the glycosylated hemoglobin (HbA1c) and $\mathrm{FBG}$ at the baseline, and 3 and 6 months. HbA1c significantly increased at 3 months, whereas FBG did not significantly change at 3 or 6 months. The current study compared all the patients and revealed a significantly increased FBG. FBG significantly increased in the orchiectomy group, but it did not significantly change in the LHRH group, without significant differences between the two groups. No significant difference was also determined between or within the antiandrogen groups.

\section{Study Limitations}

Two important limitations were determined in this study: First is its retrospective nature, and second is the relatively small number of patients.

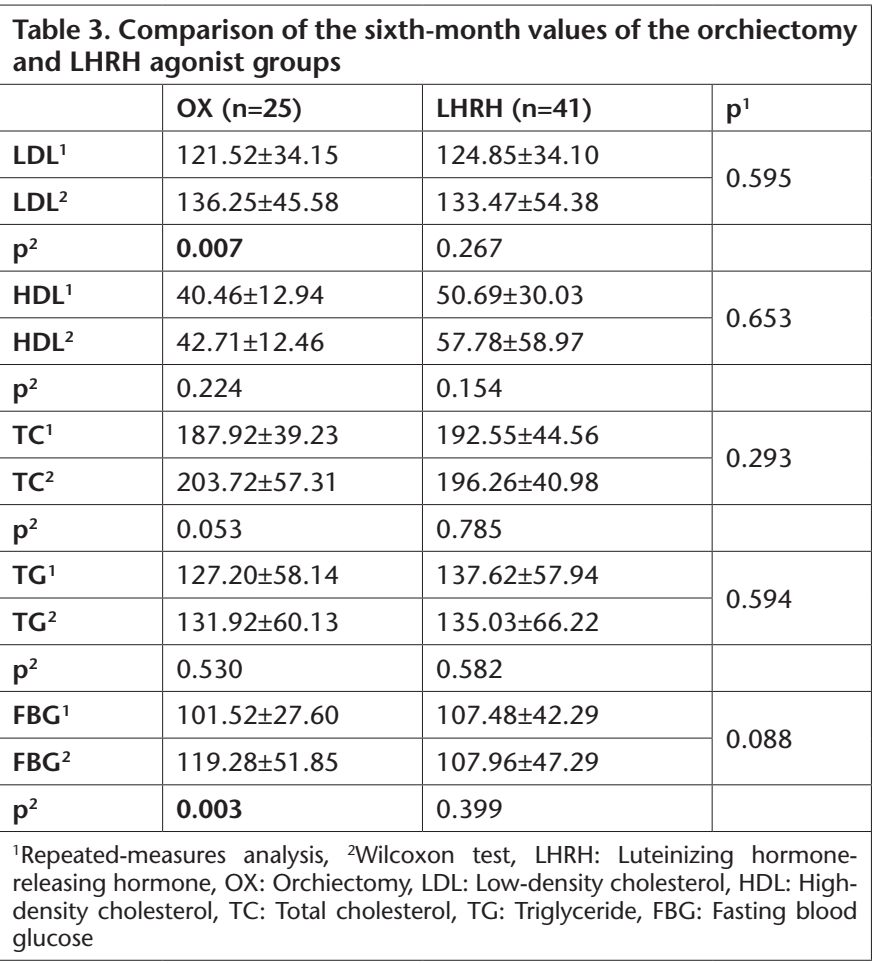




\begin{tabular}{|c|c|c|c|c|}
\hline & Cyproterone & Bicalutamide & None & $p$-value \\
\hline LDL $^{1}$ & $121.76 \pm 25.70$ & $123.00 \pm 36.61$ & $136.00 \pm 45.66$ & \multirow{2}{*}{0.160} \\
\hline $\mathrm{LDL}^{2}$ & $120.97 \pm 30.50$ & $142.36 \pm 59.78$ & $128.84 \pm 30.84$ & \\
\hline$p^{2}$ & 0.808 & $<0.001$ & 0.892 & \\
\hline $\mathrm{HDL}^{1}$ & $46.01 \pm 14.64$ & $47.08 \pm 30.84$ & $48.12 \pm 8.75$ & \multirow{2}{*}{0.330} \\
\hline $\mathrm{HDL}^{2}$ & $40.67 \pm 11.44$ & $58.47 \pm 9.52$ & $48.74 \pm 16.71$ & \\
\hline$p^{2}$ & 0.076 & $<0.001$ & 0.893 & \\
\hline $\mathrm{TC}^{1}$ & $189.48 \pm 29.70$ & $188.96 \pm 47.21$ & $211.00 \pm 49.84$ & \multirow{2}{*}{$<0.001$} \\
\hline $\mathrm{TC}^{2}$ & $179.81 \pm 39.09$ & $213.17 \pm 46.89$ & $167.42 \pm 49.48$ & \\
\hline$p^{2}$ & 0.237 & 0.001 & 0.043 & \\
\hline $\mathrm{TG}^{1}$ & $108.67 \pm 4.75$ & $142.41 \pm 56.07$ & $168.80 \pm 55.22$ & \multirow{2}{*}{0.237} \\
\hline $\mathrm{TG}^{2}$ & $94.33 \pm 48.51$ & $147.10 \pm 62.18$ & $193.84 \pm 40.80$ & \\
\hline$p^{2}$ & 0.313 & 0.757 & 0.465 & \\
\hline $\mathrm{FBG}^{1}$ & $99.05 \pm 23.55$ & $106.15 \pm 39.84$ & $123.72 \pm 60.92$ & \multirow{2}{*}{0.337} \\
\hline $\mathrm{FBG}^{2}$ & $116.52 \pm 57.37$ & $107.65 \pm 2.88$ & $131.06 \pm 62.13$ & \\
\hline$p^{2}$ & 0.135 & 0.051 & 0.043 & \\
\hline
\end{tabular}

${ }^{1}$ Repeated-measures analysis, ${ }^{2}$ Wilcoxon test, LDL: Low-density cholesterol, HDL: High-density cholesterol, TC: Total cholesterol, TG: Triglyceride, FBG: Fasting blood glucose

\section{Conclusion}

Many literature studies were reported on the lipid profile of ADT and FBG values of patients with prostate cancer. However, studies that compared different antiandrogen treatments were not determined in the changes in the lipid profile and FBG of these patients. Our results revealed a significantly increased TC value in bicalutamide compared to cyproterone. Statistically significant results would have been probably obtained in the changes in LDL and $\mathrm{HDL}$ if a higher number of patients had been evaluated. Prospective studies with a higher number of patients are necessary to clarify this issue.

\section{Acknowledgements}

Publication: The results of the study were not published in full or in part in form of abstracts.

Contribution: There is not any contributors who may not be listed as author.

Conflict of Interest: No conflict of interest was declared by the author.

Financial Disclosure: The author declared that this study received no financial support.

\section{Ethics}

Ethics Committee Approval: Approval was obtained from the Ethics Committee of Diyarbakır Gazi Yaşargil Training and Research Hospital with the date and number 772/29.05.2021.

Informed Consent: Retrospective study.

Peer-review: Externally and internally peer-reviewed.

\section{References}

1. Jemal A, Siegel R, Ward E, et al. Cancer statistics, 2007. CA Cancer J Clin 2007;57:43-66.
2. Barry MJ, Delorenzo MA, Walker-Corkery ES, et al. The rising prevalence of androgen deprivation among older American men since the advent of prostate-specific antigen testing: a populationbased cohort study. BJU Int 2006;98:973-978.

3. Meng MV, Grossfeld GD, Sadetsky N, et al. Contemporary patterns of androgen deprivation therapy use for newly diagnosed prostate cancer. Urology 2002;60(3 Suppl 1):7-11.

4. Loblaw DA, Virgo KS, Nam R, et al. Initial hormonal management of Androgen-sensitive metastatic, recurrent, or progressive prostate cancer: 2006 Update of an American society of clinical oncology practice guideline. J Clin Oncol 2007;25:1596-1605.

5. Isbarn H, Boccon-Gibod L, Carroll PR, et al. Androgen deprivation therapy for the treatment of prostate cancer: consider both benefits and risks. Eur Urol 2009;55:62-75.

6. Schwandt A, Garcia JA. Complications of androgen deprivation therapy in prostate cancer. Curr Opin Urol 2009;19:322-326.

7. Taylor LG, Canfield SE, Du XL. Review of major adverse effects of androgen-deprivation therapy in men with prostate cancer. Cancer 2009; 115:2388-2399.

8. Keating NL, O'Malley AJ, Smith MR. Diabetes and cardiovascular disease during androgen deprivation therapy for prostate cancer. J Clin Oncol 2006;24:4448-4456.

9. Tadros NN, Garzotto M. Androgen deprivation therapy for prostate cancer: not so simple. Asian J Androl 2011;13:187-188.

10. Brown BW, Brauner C, Minnotte MC. Noncancer deaths in white adult cancer patients. J Natl Cancer Inst 1993;85:979-987.

11. Keating NL, O'Malley AJ, Freedland SJ, Smith MR. Diabetes and cardiovascular disease during androgen deprivation therapy: observational study of veterans with prostate cancer. J Natl Cancer Inst 2010;102:39-46.

12. Bo JJ, Zhang C, Zhang LH, et al. Androgen deprivation therapy through bilateral orchiectomy: increased metabolic risks. Asian J Androl 2011;13:833-837.

13. Morote J, Gómez-Caamaño A, Alvarez-Ossorio JL, et al. The metabolic syndrome and its components in patients with prostate cancer on androgen deprivation therapy. J Urol 2015;193:1963-1969.

14. Gagliano-Jucá T, Burak MF, Pencina KM, et al. Metabolic Changes in Androgen-Deprived Nondiabetic Men With Prostate Cancer Are Not Mediated by Cytokines or aP2. J Clin Endocrinol Metab 2018;103:3900-3908.

15. Yannucci J, Manola J, Garnick MB, et al. The effect of androgen deprivation therapy on fasting serum lipid and glucose parameters. I Urol 2006;176:520-525.

16. Braga-Basaria M, Muller DC, Carducci MA, et al. Lipoprotein profile in men with prostate cancer undergoing androgen deprivation therapy. Int J Impot Res 2006;18:494-498.

17. Dockery F, Bulpitt CJ, Agarwal S, et al. Testosterone suppression in men with prostate cancer leads to an increase in arterial stiffness and hyperinsulinaemia. Clin Sci (Lond) 2003;104:195-201.

18. Nishiyama T, Ishizaki F, Anraku T, et al. The influence of androgen deprivation therapy on metabolism in patients with prostate cancer. J Clin Endocrinol Metab 2005;90:657-660.

19. Smith MR, Finkelstein JS, McGovern FJ, et al. Changes in body composition during androgen deprivation therapy for prostate cancer. J Clin Endocrinol Metab 2002;87:599-603.

20. Smith JC, Bennett S, Evans LM, et al. The effects of induced hypogonadism on arterial stiffness, body composition, and metabolic parameters in males with prostate cancer. J Clin Endocrinol Metab 2001;86:4261-4267.

21. Wolny-Rokicka E, Tukiendorf A, Wydmański J, et al. Lipid Status During Combined Treatment in Prostate Cancer Patients. Am J Mens Health 2019;13:1557988319876488.

22. Chen KC, Peng CC, Hsieh HM, et al. Antiandrogenic therapy can cause coronary arterial disease. Int J Urol 2005;12:886-891. 
23. Paisey RB, Kadow C, Bolton C, et al. Effects of cyproterone acetate and a long-acting LHRH analogue on serum lipoproteins in patients with carcinoma of the prostate. J R Soc Med 1986;79:210-211.

24. Wallentin L, Varenhorst E. Plasma lipoproteins during treatment with cyproterone acetate in men with prostatic carcinoma. J Clin Endocrinol Metab 1980;51:1118-1122.

25. Salvador C, Planas J, Agreda F, et al. Analysis of the lipid profile and atherogenic risk during androgen deprivation therapy in prostate cancer patients. Urol Int 2013;90:41-44.

26. Sağlam HS, Köse O, Kumsar S, et al. Fasting blood glucose and lipid profile alterations following twelve-month androgen deprivation therapy in men with prostate cancer. ScientificWorldjournal 2012;2012:696329.
27. Stellato RK, Feldman HA, Hamdy O, et al. Testosterone, sex hormonebinding globulin, and the development of type 2 diabetes in middleaged men: prospective results from the Massachusetts Male Aging Study. Diabetes Care 2000;23:490-494.

28. Simon D, Charles MA, Nahoul K, et al. Association between plasma total testosterone and cardiovascular risk factors in healthy adult men: the Telecom Study. J Clin Endocrinol Metab 1997;82:682-685.

29. Ding EL, Song Y, Malik VS, Liu S. Sex differences of endogenous sex hormones and risk of type 2 diabetes: a systematic review and metaanalysis. JAMA 2006;295:1288-1299. 